\title{
American Society of Clinical Oncology 2015- Highlights of Non-colorectal Gastrointestinal Cancers
}

\author{
Eileen M O'Reilly, MD
}

Medical Oncologist and Associate Director for Clinical Research, David M Rubenstein Center for Pancreatic Cancer Research, Memorial Sloan Kettering Cancer Center, New York, New York, us

DOI: http://doi.org/10.17925/OHR.2015.11.02.137

\begin{abstract}
This editorial covers the key highlights on Non-Colorectal Gastrointestinal Cancers from American Society of Clinical Oncology Annual Meeting 2015.

Keywords

Non-colorectal gastrointestinal cancers, pembrolizumab, PD-1 inhibitor, KEYNOTE-012, RILOMET-1 trial, rilotumumab, erlotinib, PEGPH20

Disclosure: Eileen M O'Reilly, MD, has nothing to declare in relation to this article. No funding was received in the publication of this article.

open Access: This article is published under the Creative Commons Attribution Noncommercial License, which permits any noncommercial use, distribution, adaptation and reproduction provided the original author(s) and source are given appropriate credit.

Received: August 25, 2015 Published Online: November 10, 2015 Citation: Oncology \& Hematology Review, 2015;11(2):137-8

Correspondence: Eileen M O'Reilly, MD, Memorial Sloan Kettering Cancer Center, 300 East 66th Street, New York, NY 10065, US. E: oreillye@mskcc.org
\end{abstract}

The 2015 annual American Society of Clinical Oncology meeting brought refinements rather than major practice changes to the field of noncolorectal gastrointestinal (GI) cancers, with one notable exception and that is for the emerging endorsement of the role of immune therapies in these diseases. Arguably the most important observation from the meeting overall was the recognition of a strong signal for pembrolizumab (MK3475), a programmed death-1 (PD-1) antibody inhibitor, dosed at $10 \mathrm{mg} / \mathrm{kg}$ intravenously every 2 weeks, in malignancies with microsatellite instability (MSI-high) in both colorectal cancers and other non-colorectal cancers (non$\mathrm{CRC}$ ), and showed markedly favorable activity in the patients with MSI-high compared to no significant activity in patients with MSI-stable tumors. ${ }^{1}$ Very specifically, in the subset of DNA mismatch repair protein-deficient, nonCRC ( $n=10$, including, ampullary, biliary, small bowel, gastric cancers), the response rate was $60 \%$ and the disease control rate was $70 \%$. About $40 \%$ of these patients had hereditary non-polyposis colorectal cancer. While the subset of patients with non-CRC GI cancers with MSI-high status is likely to be small, single digit percent of all-comers, the results in these patients are dramatic. The implication for practice is to consider mismatch repair protein expression via immunohistochemical $(\mathrm{IHC})$ evaluation or polymerase chain reaction evaluation of DNA microsatellite instability in these patients and if mismatch repair deficiency (MMR)-deficient/MSI-high consider enrollment in a PD-1 or programmed death-ligand 1 (PD-L1) antibody trial to fully understand how and when these agents should be utilized.

Continuing the theme of immune therapy, the update from the KEYNOTE-012 single agent evaluation of pembrolizumab in previously treated metastatic gastric cancer further highlighted the enthusiasm for immune therapy strategies in $\mathrm{Gl}$ cancers. ${ }^{2}$ The rationale for their use is compelling based on subsets of patients with high somatic mutational burden in gastric cancer, ${ }^{3}$ the association of PD-1 and PD-L1 with a poor prognosis and results from the Cancer Genome Atlas Research Network suggesting that certain subsets of patients with gastric cancer with Epstein-Barr virus and MSI are more likely to benefit from immune therapies. ${ }^{4}$ In this singlearm non-randomized evaluation of pembrolizumab, Bang and colleagues evaluated patients who had had at least two lines of prior therapy and a good functional status and for eligibility required PD-L1 expression by $\mathrm{IHC}$ in $\geq 1 \%$ of tumor cells or stroma. For the primary endpoint of response, there was a $22 \%$ objective response rate to pembrolizumab in gastric cancer with significant durability with a median of 40 weeks, although in some patients, the durability extended to a year and beyond, and with an overall survival of 11.4 months. A detailed analysis of PDL1 expression and outcome suggested that an improved benefit was observed in those with greater PD-L1 expression. A similar response signal was observed in KEYNOTE-028 in an esophageal cohort. There are now a series of immune therapy trials in advanced esophagogastric malignancies including an evaluation of pembrolizumab in a third-line setting, along with an evaluation with standard platinum/frontline fluoropyrimidine therapy, a comparison to paclitaxel in a second-line setting, an evaluation as a single agent in PD-L1 positivity frontline, and with other agents evaluating the addition of a PD-1 antibody with/without anti-cytotoxic T-lymphocyteassociated protein 4 (anti-CTLA4) therapy. The next few years will more definitively clarify the disease stages and settings for optimal utility from immune therapies in esophagogastric malignancies.

On a different theme, this year's meeting has likely closed an era/strategy of development in esophagogastric malignancies with the final results of two 
MET-targeting phase III trials not showing a significant benefit. Cunningham and colleagues ${ }^{5}$ presented the results of the RILOMET- 1 trial, a randomized phase III study evaluating the addition of rilotumumab, a monoclonal antibody against the ligand of the MET receptor, hepatocyte growth factor, or placebo, combined with epirubicin, cisplatin, and capecitabine (ECX) in untreated advanced esophagogastric cancers. The primary endpoint of overall survival was 9.6 (confidence interval [Cl] 7.9-11.4) months in the experimental arm and $11.5(\mathrm{Cl} 9.7-13.1)$ months in the control arm, hazard ratio 1.36 (1.05-1.75); $p=0.021$, inferring a worse outcome related to higher rates of disease progression in the experimental arm. Similarly, even in the highest MET positive subgroup by IHC evaluation, there was no association with either worse prognosis or better outcome for the addition of rilotumumab. Parallel results were seen in a related phase III trial (METGastric) evaluating the addition of onartuzumab (anti-MET antibody) to FOLFOX (folinic acid, fluorouracil, oxaliplatin), with no significant benefit observed for the addition of antibody over standard therapy. ${ }^{6}$ Collectively these results are disappointing and suggest that further evaluation of MET targeting strategies has limited utility in these diseases.

In pancreas malignancies, the results of a phase III adjuvant trial (CONKO-005) evaluating the addition of erlotinib to standard therapy, gemcitabine, showed no significant improvement in overall survival. ${ }^{7}$ These results are in line with recently reported results from the LAP-07 trial evaluating erlotinib in locally advanced, inoperable pancreas cancer, where the addition of erlotinib did not add to gemcitabine in terms of the primary endpoint of overall survival. Collectively these results underscore the very finite and limited utility, if any, of erlotinib in earlier stage pancreas adenocarcinoma, although the continued elusive identification of a biomarker selected subset could possibly change that interpretation. On a more positive note, Hingorani and colleagues evaluated the addition of PEGPH2O, a pegylated hyaluronidase enzyme in patients with untreated metastatic pancreas adenocarcinoma. ${ }^{8}$ In a subset of patients with high levels of hyaluronan $(\mathrm{HA})$, a key stromal component, a significant survival signal was observed for the addition of PEGPH2O to a cytotoxic backbone of gemcitabine and nab-paclitaxel over the chemotherapy regimen alone. Initially an increased thromboembolic signal was observed in the experimental arm; however, this appeared to be mitigated by a strategy of careful patient selection and primary prophylaxis with low molecular weight heparin. Following on, a phase III trial evaluating the addition of PEGPH20/placebo to gemcitabine and nab-paclitaxel in a high-HA subgroup of patients with untreated metastatic pancreas adenocarcinoma is scheduled to commence in 2016 to more definitively evaluate this preliminary signal.

To sum up, the conclusions from the 2015 American Society of Clinical Oncology meeting were evolutionary rather than revolutionary. On a forward note, we now have conclusive evidence that non-colorectal Gl cancers can benefit from immune therapies. In pancreatic adenocarcinoma, the search for biomarkers continues with HA possibly representing one such biomarker and for which phase III investigation is planned. Despite initial promise, anti-MET antibody targeting strategies in esophagogastric cancers will not be further developed and the field looks to the promise of other approaches including novel strategies for targeting human epidermal growth factor receptor 2, evaluation of stem cell-targeting agents, and the potential for PD1 and related immune options.
1. Le DT, Uram JN, Wang H, et al., PD-1 blockade in tumors with mismatch repair deficiency, ASCO Meeting Abstracts, 2015;33:LBA100.

2. Bang Y-J, Chung H-C, Shankaran V, et al., Relationship between PD-L1 expression and clinical outcomes in patients with advanced gastric cancer treated with the anti-PD-1 monoclonal antibody pembrolizumab (MK-3475) in KEYNOTE-012, ASCO antibody pembrolizumab (MK-3475)

3. Alexandrov LB, Nik-Zainal S, Wedge DC, et al., Signatures of mutational processes in human cancer, Nature, 2013;500:415-21.

4. Cancer Genome Atlas Research Network, Comprehensive molecular characterization of gastric adenocarcinoma, Nature
2014;513:202-9.

5. Cunningham D, Tebbutt NC, Davidenko I, et al., Phase III, randomized, double-blind, multicenter, placebo (P)-controlled trial of rilotumumab (R) plus epirubicin, cisplatin and capecitabine (ECX) as first-line therapy in patients (pts) with advanced MET-positive (pos) gastric or gastroesophageal junction (G/GEJ) cancer: RILOMET-1 study, ASCO Meeting Abstracts, 2015:33:4000

6. Shah MA, Bang Y-J, Lordick F, et al., METGastric: a phase III study of onartuzumab plus mFOLFOX6 in patients with metastatic HER2-negative (HER2-) and MET-positive (MET+) adenocarcinoma of the stomach or gastroesophageal junction
(GEC), ASCO Meeting Abstracts, 2015;33:4012.

7. Sinn M, Liersch T, Gellert K, et al., CONKO-005: adjuvant therapy in RO resected pancreatic cancer patients with gemcitabine plus erlotinib versus gemcitabine for 24 weeks--A prospective randomized phase III study, ASCO Meeting Abstracts, 2015;33:4007.

8. Hingorani SR, Harris WP, Hendifar AE, et al., High response rate and PFS with PEGPH2O added to nab-paclitaxel/gemcitabine in stage IV previously untreated pancreatic cancer patients with high-HA tumors: interim results of a randomized phase ॥ study, 2015 ASCO Annual Meeting. Abstract 4006. Presented May $31,2015$. 